\title{
NUMERICAL STUDY OF MAGNETIC PARTICLES CONCENTRATION IN BIOFLUID (BLOOD) UNDER THE INFLUENCE OF HIGH GRADIENT MAGNETIC FIELD IN MICROCHANNEL
}

\author{
V. C. Loukopoulos ${ }^{1}$, G. C. Bourantas ${ }^{2}$, D. Labropoulos ${ }^{3}$, V. M. G. Nikiforidis ${ }^{3}$ S. P. A. \\ Bordas $^{2,4,5}$ and G. C. Nikiforidis ${ }^{3}$ \\ ${ }^{1}$ Department of Physics, University of Patras \\ Patras, GR 26500, Rion, Greece \\ e-mail: vxloukop@physics.upatras.gr \\ ${ }^{2}$ Faculty of Science, Technology and Communication, University of Luxembourg \\ Campus Kirchberg, 6 rue Richard Coudenhove-Kalergi L-1359, Luxembourg \\ \{georgios.bourantas,stephane.bordas\}@ uni.lu \\ ${ }^{3}$ Department of Medical Physics, School of Medicine, University of Patras \\ GR 26504,Rion, Greece \\ dimlab@upatras.gr,martin-nikif@hotmail.com,gnikif@med.upatras.gr \\ ${ }^{4}$ School of Engineering, Cardiff University \\ The Parade, CF24 3AA Cardiff, United Kingdom \\ ${ }^{5}$ Intelligent Systems for Medicine Laboratory, School of Mechanical and Chemical Engineering, The \\ University of Western Australia \\ Stirling Highway, Crawley/Perth, WA 6009, Australia
}

Keywords: Biomagnetic Separation, Drug Delivery, Hyperthermia Treatment, Non Newtonian, Two-phase Flow, Magnetophoretic Mass Transfer; Molecular Diffusion, Meshless.

Abstract. A meshless numerical scheme is developed in order to simulate the magnetically mediated separation of biological mixture used in lab-on-chip devices as solid carriers for capturing, transporting and detecting biological magnetic labeled entities, as well as for drug delivering, magnetic hyperthermia treatment, magnetic resonance imaging, magnetofection, etc. Due to the imposed magnetic field stagnation regions are developed, leading to the accumulation of the magnetic labeled species and finally to their collection from the heterogeneous mixture. The role of (i) the intensity of magnetic field and its gradient, (ii) the position of magnetic field, (iii) the magnetic susceptibility of magnetic particles, (iv) the volume concentration of magnetic particles and their size, (v) the flow velocity in the magnetic fluidic interactions and interplay between the magnetophoretic mass transfer and molecular diffusion are thoroughly investigated. Both Newtonian and non-Newtonian blood flow models are considered. 


\section{INTRODUCTION}

Biomagnetic fluid dynamics (BFD) investigates the dynamics of biological fluids under the presence of magnetic field. BFD accounts in numerous bioengineering applications [1-3] and there are a number of mathematical models proposed regarding biomagnetic fluid flow under the action of an applied magnetic field.

The governing equations for incompressible fluid flow are similar to those used in ferrohydrodynamics (FHD). In [3, 4] the viscous, steady, two-dimensional, incompressible, laminar biomagnetic fluid (blood) flow between two parallel flat plates (channel) was studied, with the fluid considered as homogeneous. Moreover, magnetic fluids are used in medicine, especially in high-gradient magnetic separation (HGMS), magnetic drug targeting (MDT) and ferrofluid sealing [5]. The flow model in [2-4], assumes that suspended particles and base fluid constitute a stable colloidal suspension with infinitely strong coupling, in which the particles are not amenable to re-distribution or drifting relative to the base fluid under the effect of an external magnetic force. Thus, the fluid-particles mixture interacts with the magnetic force as a whole fluid. On the other hand, the model employed in [5-9], accommodates magnetophoretic drifting relative to the base fluid and therefore allows particles re-distribution when an advection-diffusion particle concentration is coupled with the Navier-Stokes equations. In comparison, the traditional BFD and FHD studies treat the volumetric magnetic body force as simply that of a single-phase flow, while the [5-9] studies treat it as of a two-phase flow where each phase can exhibit its distinctive magnetic response. One-way particle-fluid coupling approach (Eulerian) was adopted in [5-8] and two-way particle-fluid coupling approach (Lagrangian-Eulerian) in [9].

In the present study we investigate a two phase flow of a non-Newtonian biomagnetic fluid between two horizontal parallel plates, under the action of a non uniform magnetic field and we examine the role of $(i)$ the intensity of magnetic field and its gradient, ( $i i)$ the position of magnetic field, (iii) the magnetic susceptibility of magnetic particles, (iv) the volume concentration of magnetic particles (nanoparticles) and their size, and $(v)$ the flow velocity.

\section{EQUATIONS AND MATHEMATICAL MODEL}

A non-uniform magnetic field is applied to microchannel flow containing magnetically tagged microspheres. A two-dimensional rectangle microchannel is considered with width $(W)$ and length $(L)$ equal to $1 \mathrm{~mm}$ and $20 \mathrm{~mm}$ respectively. Assumptions as in $[6,7]$ are adopted in the present study.

The governing dimensional equations of the isothermal fluid flow in $x$ - and $y$-direction are as follows:

$$
\begin{aligned}
& \frac{\partial u}{\partial x}+\frac{\partial v}{\partial y}=0 \\
& \rho\left(u \frac{\partial u}{\partial x}+v \frac{\partial u}{\partial y}\right)=-\frac{\partial p}{\partial x}+\mu\left(\frac{\partial^{2} u}{\partial x^{2}}+\frac{\partial^{2} u}{\partial y^{2}}\right)+F_{x} \\
& \rho\left(u \frac{\partial v}{\partial x}+v \frac{\partial v}{\partial y}\right)=-\frac{\partial p}{\partial y}+\mu\left(\frac{\partial^{2} v}{\partial x^{2}}+\frac{\partial^{2} v}{\partial y^{2}}\right)+F_{y}
\end{aligned}
$$

where $F_{x}$ and $F_{y}$ are the volume forces in the $x$ - and $y$-directions, respectively and represent the effect of magnetic particles concentration, while $\rho$ and $\mu$ are the density and the viscosity 
of the biofluid (blood). Magnetic particles are considered as super-paramagnetic material without permanent magnetization at room temperature. Velocity components in the $x$ - and $y$ direction are defined by $u$ and $v$, respectively. The volume forces $\left(F_{x}\right.$ and $\left.F_{y}\right)$ are equal to the magnetic forces applied on a single particle $\left(F_{m a g, x}\right.$ and $\left.F_{\text {mag, } y}\right)$, multiplied by the number of particles per unit volume $\left(F_{x}=n_{p}(x, y) F_{\text {mag, } x}\right.$ and $\left.F_{y}=n_{p}(x, y) F_{\text {mag, } y}\right)$. They are defined as [5-8]:

$$
\boldsymbol{F}_{\text {mag }}=\frac{1}{2} V_{p} \mu_{0} \chi \nabla H^{2}
$$

with $H, \mu_{0}$ and $\chi$ being the external magnetic field intensity, the magnetic permeability of vacuum and the magnetic susceptibility of the particles, respectively ( $\mathrm{p}$ subscript refers to particles). The dimensionless concentration $\left(C(x, y)=n_{p}(x, y) / n_{0}\right)$ is defined with respect to the reference particle density $\eta_{0}$ at the flow entrance. In addition, the non-dimensional volume concentration $(C)$ is written as [5-8]

$$
\nabla\left(C v_{p}\right)=\nabla(D \nabla C) \text {. }
$$

In Eq. (5), $D$ is the diffusion coefficient and the velocity of particles $\boldsymbol{v}_{p}$ is calculated by balancing the hydrodynamic and magnetic forces $[6,7]$

$$
\boldsymbol{v}_{p}=\boldsymbol{v}_{f}+\boldsymbol{v}_{\text {mag }}
$$

where $\boldsymbol{v}_{\text {mag }}=\boldsymbol{F}_{\text {mag }} / 6 \pi \mu r_{p}$ is given by Stokes drag law, $\boldsymbol{v}_{f}=u \boldsymbol{i}+v \boldsymbol{j}$ is the velocity of the fluid computed by Eqs. (1) and (2) and, $r_{p}$ is the particle radius.

The boundary conditions are defined as:

- for the velocity components: at inlet fully developed flow is considered $(u=u(y)$, $v=0)$, no-slip boundary conditions are imposed on the upper and lower wall and fully developed flow at the outlet $(u, x=0, v=0)$.

- for the dimensionless concentration: concentration $C_{i n}=1$ at the inlet, insulated on the upper and lower walls and, convection at the outlet.

Since the viscosity of a non-Newtonian fluid (blood) is a function of shear rate, a relation between the viscosity and the shear rate is required, (shear-thinning viscosity of the blood). The relation between the shear stress $\tau$ and the shear rate $\dot{\gamma}$ is as $\tau=\mu[\dot{\gamma}]$. The viscosity is given by the power law model $\mu=m \dot{\gamma}^{n-1}[10,11]$.

\section{RESULTS AND DISCUSSION}

\subsection{Verification of the numerical results and grid independent test}

A Meshless Point Collocation (MPCM) scheme along with the Moving Least Squares (MLS) approximation method is applied for the numerical solution of the governing equations. An iterative scheme, developed and presented in [12], was used to numerically solve the velocity-vorticity formulation of the flow equations. Mass conservation (continuity equation) was ensured enforcing a velocity-correction scheme. The accuracy of the proposed scheme was verified against the numerical results obtained by a Meshless Point Collocation method (MPCM) along with the IPOT (Implicit Potential) and DCP SE (Discretization Correction Particle Strength Exchange) approximation for the field variables for the numerical solution 
of the model equations in the velocity-pressure formulation [13], Fig. 1. The magnetic field intensity is $1 \times 10^{6} \mathrm{~A} \mathrm{~m}^{-1}$. The base fluid is blood with density $800 \mathrm{~kg} \mathrm{~m}^{-3}$ [8] and viscosity given by the power law ( $m=0.035$ and $n=0.6[10,11]$ ), in Poise unit. We assume that the target biological entity and the attached magnetic particles constitute a single microspherical particle with $2 \mu \mathrm{m}$ radius [6-8]. The effective magnetic susceptibility $\chi$ (relative to that of magnetic particles) is equal to 0.004 , volume fraction $\varphi$ is equal to 0.03 , the mean inlet velocity is $0.001 \mathrm{~m} \mathrm{~s}^{-1}$ (Plug flow) and the Particle diffusivity $D$ is $1 \times 10^{-9} \mathrm{~m}^{2} \mathrm{~s}^{-1}$.

An extensive mesh-refinement procedure has been conducted, in order to ensure a gridindependent numerical solution. The numerical results presented are obtained using a $31 \times 20 \times 31$ uniform nodal distribution.

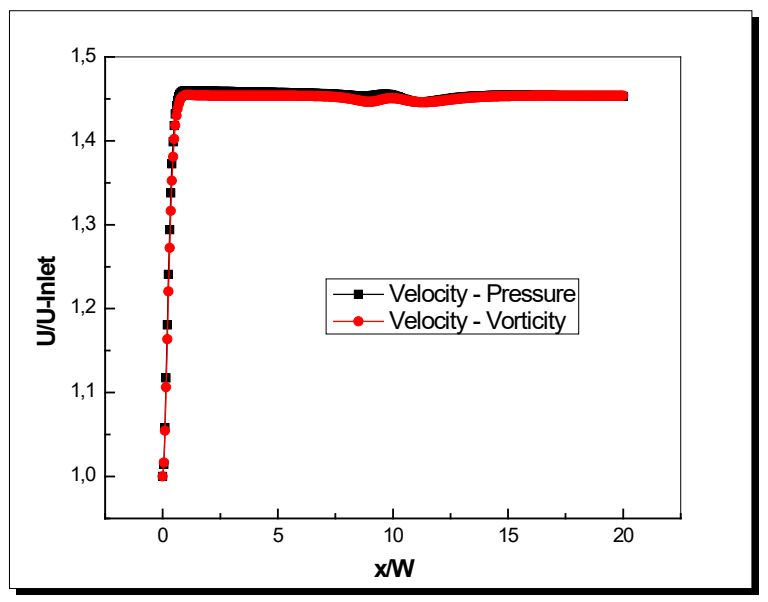

Figure 1: Verification.

\subsection{Discussion}

Figure 2 depicts velocity magnitude of the Newtonian and non-Newtonian fluid (blood mixture) along the channel central line, considering mean velocity inlet, magnetic particles diameter and magnetic susceptibility equal to $0.001 \mathrm{~m} \mathrm{~s}^{-1}, 2 \mu \mathrm{m}$ and 0.004 , respectively.

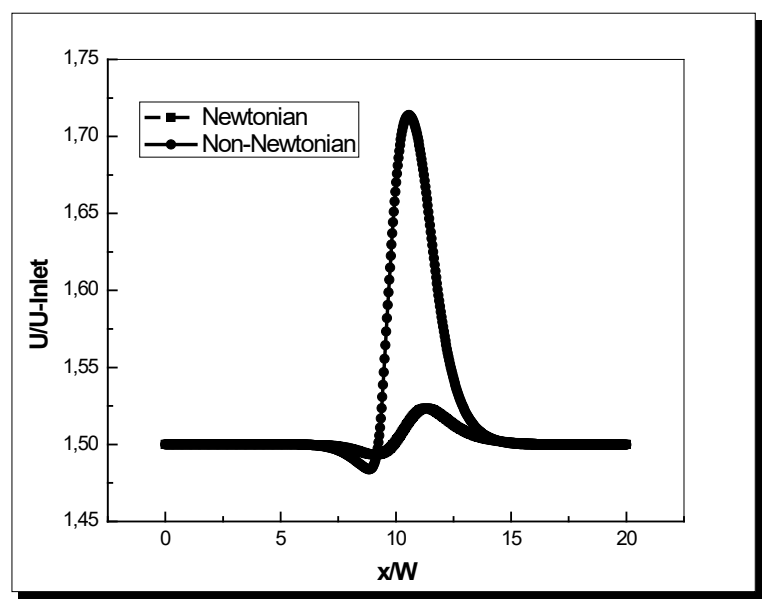

Figure 2: Non-dimensional velocity profile at the central line of the channel for Newtonian and nonNewtonian behavior of the magnetic fluid (blood) mixture. 
Figure 3 shows the stream function and the particles concentration contours for mean inlet velocity equal to $0.001 \mathrm{~m} \mathrm{~s}^{-1}$, magnetic particles diameter $2 \mu \mathrm{m}$ and magnetic susceptibility 0.004 , considering two different positions for the magnetic source, that is, $b=4$ and $3 \mathrm{~mm}$. We can observe that a secondary vortex close to the upper plate is formed when $b=4 \mathrm{~mm}$, while a secondary vortex close to the lower plate is formed when $b=3 \mathrm{~mm}$.
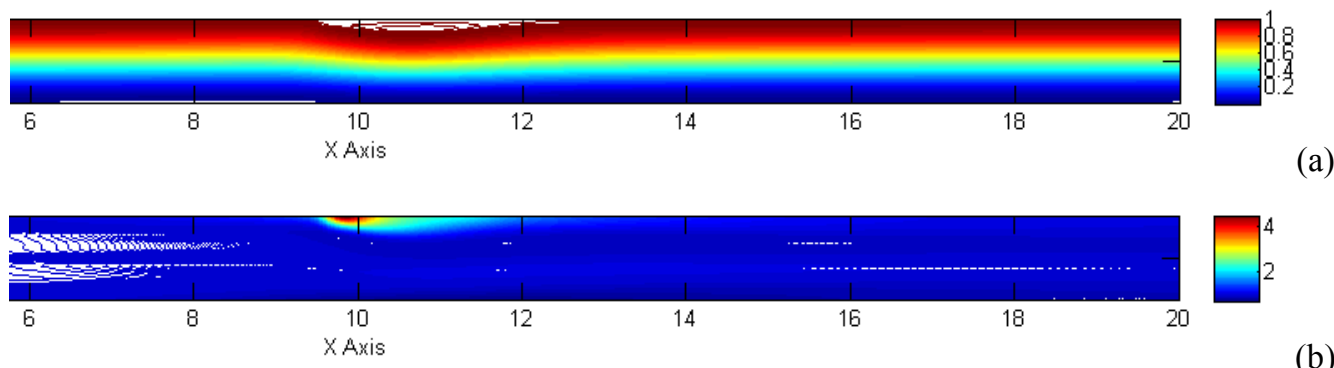

(a)

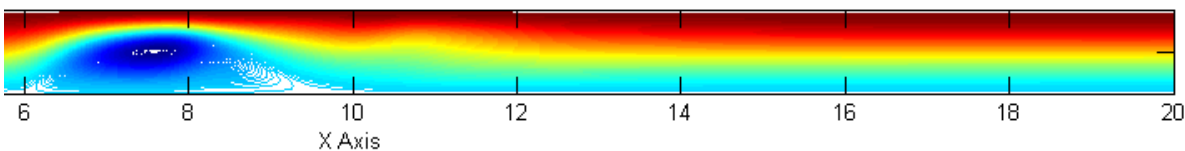

(b)

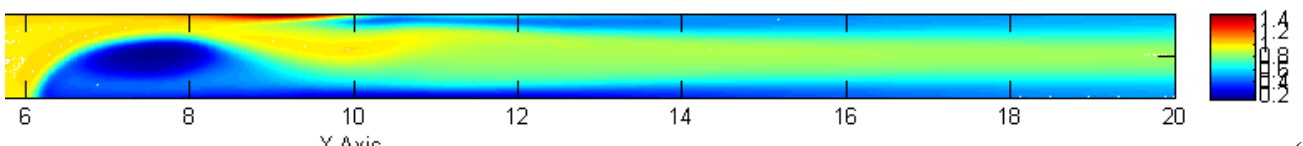

(d)

Figure 3: Stream function contours and concentration contours for mean velocity inlet, magnetic particles diameter and magnetic susceptibility equal to $0.001 \mathrm{~ms}^{-1}, 2 \mu \mathrm{m}$ and 0.004 when $b=4(\mathrm{a}, \mathrm{b})$ and $3 \mathrm{~mm}(\mathrm{c}, \mathrm{d})$.

In order to investigate the performance of the two-phase fluid flow (blood and solid magnetic particles), computations have been carried out for different parameter values. Blood viscosity values were given by the power law.

Figure 4 shows the variation of the dimensionless particle concentration at $y=0.97 \mathrm{~mm}$ (close to the upper plate) along $x$ - direction $(0 \mathrm{~mm}, 20 \mathrm{~mm})$ according to $(i)$ the intensity of magnetic field and its gradient, (ii) the position of magnetic field, (iii) the magnetic susceptibility of magnetic particles, (iv) the volume concentration of magnetic particles (nanoparticles) and their size, $(v)$ the flow velocity in the magnetic fluidic interactions, while the interplay between the magnetophoretic mass transfer and molecular diffusion is also investigated.
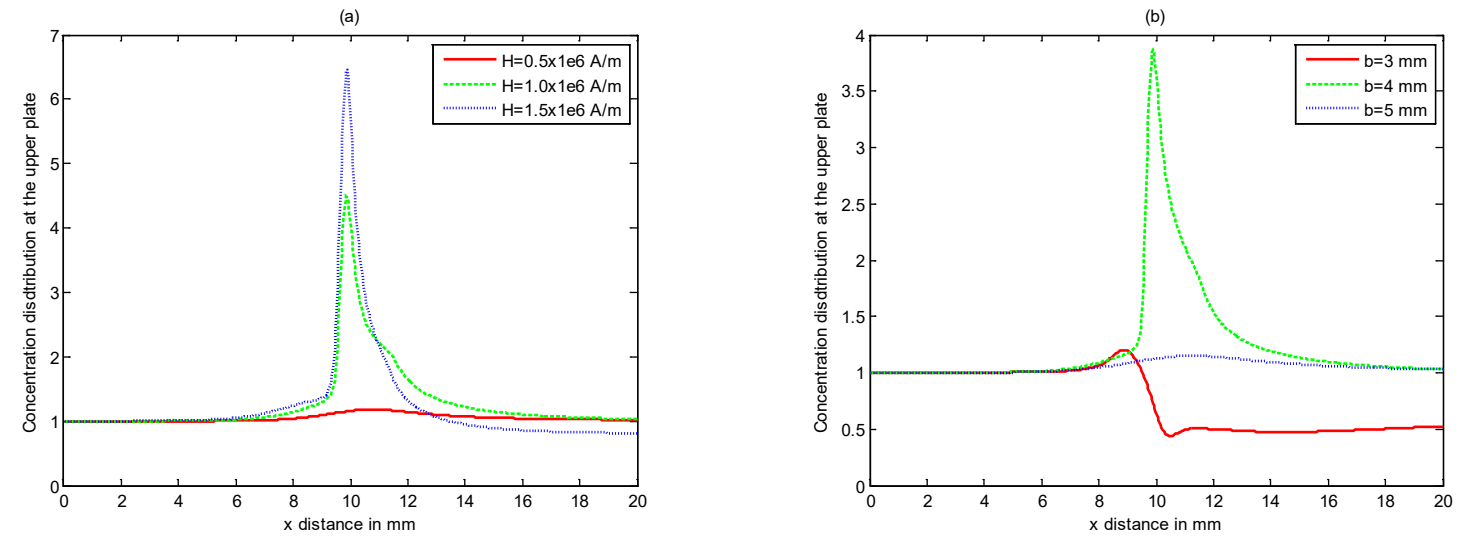

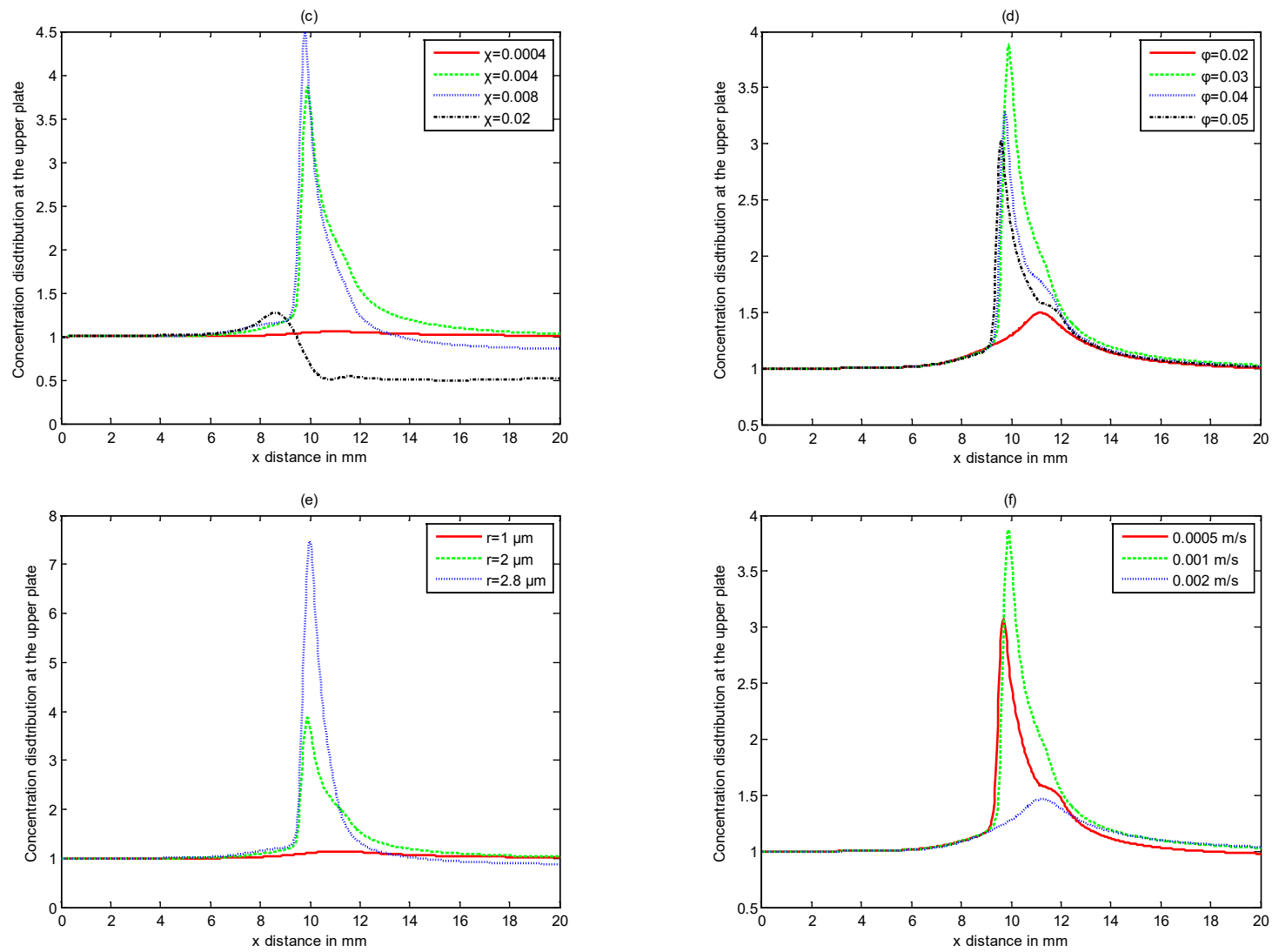

Figure 4: The variation of the dimensionless particle concentration at $y=0.97 \mathrm{~mm}$ (close to the upper plate) along $x$ direction $(0 \mathrm{~mm}, 20 \mathrm{~mm}$ ) according to (a) the intensity of magnetic field and its gradient, (b) the position of magnetic field, (c) the magnetic susceptibility of magnetic particles, (d) the volume concentration of magnetic particles (nanoparticles), (e) the size of magnetic particles, (f) the flow velocity.

Figure $4 \mathrm{a}$ presents the variation of the dimensionless particle concentration according to the intensity of magnetic field and its gradient. The base fluid is assumed to be blood with density $800 \mathrm{~kg} \mathrm{~m}^{-3}[8]$ and viscosity computed from the power law ( $m=0.035$ and $\left.n=0.6[11]\right)$, in Poise. The target biological entity and the attached magnetic particles consist a single micro-spherical particle with $2 \mu \mathrm{m}$ radius [8]. The effective magnetic susceptibility $\chi$ (relative to that of magnetic particles) is equal to 0.004 , the volume fraction $\varphi$ is equal to 0.03 and the mean inlet velocity is equal to $0.001 \mathrm{~m} \mathrm{~s}^{-1}$. The center of the permanent magnet is located at the midpoint of the plate $(a=10 \mathrm{~mm}), 4 \mathrm{~mm}$ far from the lower plate $(b=4 \mathrm{~mm})$. Particle diffusivity $D$ is assumed $1 \times 10^{-9} \mathrm{~m}^{2} \mathrm{~s}^{-1}$. For the calculations presented in Fig. 4, density, viscosity and particle diffusivity are constants. As the magnetic field intensity increases $\left(0.5 \times 10^{6} \mathrm{~A} \mathrm{~m}^{-1}, 1 \times 10^{6} \mathrm{~A} \mathrm{~m}^{-1}, 2 \times 10^{6} \mathrm{~A} \mathrm{~m}^{-1}\right)$, the concentration increases near the point $(10 \mathrm{~mm}$, $1 \mathrm{~mm}$ ) with the maximum value of the magnetic field for the flow field domain concentration. This means that increasing the intensity of the magnetic field the attractive magnetic force is dominant (magnetophoresis) over its counter tendency to diffuse upward into regions with less concentration, which eventually results to particle redistribution on the close vicinity of the point with highest magnetic strength.

In order to understand the flow behavior and the concentration contribution with varying position of the magnetic field, the virtual source is placed at $3 \mathrm{~mm}, 4 \mathrm{~mm}$ and $5 \mathrm{~mm}$ above the midpoint of the lower plate, as shown in Fig. 4b. It can be seen that the concentration increas- 
es as the magnetic source is moving towards the microchannel, until the point where the flow profile is changed. Following, the vortex formed close to the upper plate disappears and a new vortex forms close to the lower plate, as in Fig. 3c. For the computations the magnetic field intensity is $1 \times 10^{6} \mathrm{~A} \mathrm{~m}^{-1}$.

Figure $4 \mathrm{c}$ presents the variation of the dimensionless particle concentration according to the magnetic susceptibility of magnetic particles. The source is positioned at $(10 \mathrm{~mm}, 4 \mathrm{~mm})$ and the magnetic field intensity is $1 \times 10^{6} \mathrm{Am}^{-1}$. As the magnetic susceptibility increases $(0.0004,0.004,0.008,0.02)$, the concentration increases near the place $(10 \mathrm{~mm}, 1 \mathrm{~mm})$ where the magnetic field gets its maximum value, up to the point where the flow profile is changing and the vortex, formed close to the upper plate, disappears and a new vortex forms closer to the lower plate, as in Fig. 3c.

Figure $4 \mathrm{~d}$ presents the variation of the dimensionless particle concentration according to the volume concentration of magnetic particles (nanoparticles) for volume fraction $0.02,0.03$, 0.04 and 0.05 . Actually, it alters the number of particles per unit volume as $6 \times 10^{5} \mathrm{~mm}^{-3}$, $9 \times 10^{5} \mathrm{~mm}^{-3}, 12 \times 10^{5} \mathrm{~mm}^{-3}$ and $15 \times 10^{5} \mathrm{~mm}^{-3}$. The concentration increases as the volume concentration of magnetic particles is increased, up to a point beyond which while the vortex formed close to the upper plate is yet appeared, the concentration decreases as the volume concentration of magnetic particles is more increased.

Figure $4 \mathrm{e}$ presents the variation of the dimensionless particle concentration according to the size of magnetic particles. As the size of magnetic particles increases $1 \mu \mathrm{m}, 2 \mu \mathrm{m}$ and $2.8 \mu \mathrm{m}$, the concentration increases around the position $(10 \mathrm{~mm}, 1 \mathrm{~mm})$ where there is the maximum value of the magnetic field for the flow field domain.

Figure $4 \mathrm{f}$ presents the variation of the dimensionless particle concentration according to the flow velocity $\left(0.0005 \mathrm{~m} \mathrm{~s}^{-1}, 0.001 \mathrm{~m} \mathrm{~s}^{-1}, 0.002 \mathrm{~m} \mathrm{~s}^{-1}\right)$. The concentration increases as the flow velocity is increased until the point where the flow profile is changed and the vortex which is formed close to the upper plate is disappeared.

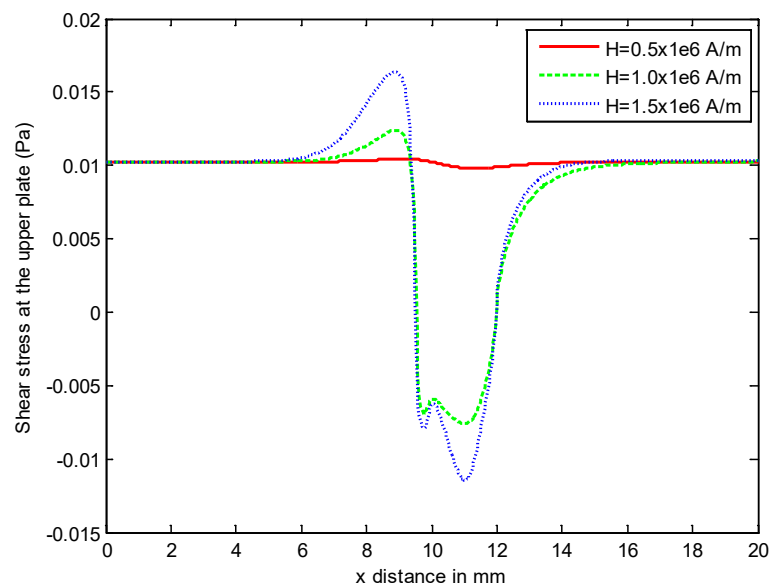

Figure 5: The variation of the dimensionless particle concentration at $y=1 \mathrm{~mm}$ along $x$ direction $(0 \mathrm{~mm}, 20$ $\mathrm{mm})$.

Figure 5 shows the variation of the shear stress magnitude along the upper plate in the micro-channel. It can be seen that high levels of shear stress are restricted to the narrow regions where the external magnetic field is applied. Peak values increase by increasing the intensity 
of magnetic field. As it mentioned in [6, 7], the fact that peak values remain far below the lytic limit implies a safe transport mechanism for the separation of biological cells.

\section{CONCLUSIONS}

- Particle size and magnetic field intensity are the two factors that mostly affect particle concentration in the vicinity of the magnetic source.

- For the position of magnetic field, the magnetic susceptibility of magnetic particles, the volume concentration of magnetic particles (nanoparticles) and the flow velocity, there is a limit, beyond which the particle concentration decreases.

- High levels of shear stress are restricted to narrow regions near the upper plate where the external magnetic field is applied. Peak values of shear stress increase by increasing the intensity of magnetic field.

\section{AKNOWLEDGMENTS}

This research has been financed by the Research Committee - University of Patras - Research Funding Program: K. Karatheodoris 2013.

\section{REFERENCES}

[1] J.M.R. Carlton, C.A. Yowell, K.A. Sturrock, J.B. Dame, Biomagnetic separation of contaminating host leukocytes from plasmodium-infected erythrocytes. Experimental Parasitology, 97, 111-114, 2001.

[2] Y. Haik, V. Pai, C.J. Chen, Development of magnetic device for cell separation. Journal of Magnetism and Magnetic Materials, 194, 254-261, 1999.

[3] V. Loukopoulos, E. Tzirtzilakis, Biomagnetic channel flow in spatially varying magnetic field. International Journal of Engineering Science, 42, 571-590, 2004.

[4] E.E. Tzirtzilakis, V.C. Loukopoulos, Biofluid flow in a channel under the action of a uniform localized magnetic field. Computational Mechanics, 36, 360-374, 2005.

[5] X.L. Li, K.L. Yao, Z.L. Liu, CFD study on the magnetic fluid delivering in the vessel in high-gradient magnetic field. Journal of Magnetism and Magnetic Materials, 320, 1753-1758, 2008.

[6] S.A. Khashan, E. Elnajjar, Y. Haik, CFD simulation of the magnetophoretic separation in a microchannel. Journal of Magnetism and Magnetic Materials, 323, 2960-2967, 2011.

[7] S.A. Khashan, E. Elnajjar, Y. Haik, Numerical simulation of the continuous biomagnetic separation in a two-dimensional channel. International Journal of Multiphase Flow, 37, 947-955, 2011.

[8] M. R. Habibi, M. Ghasemi, Numerical study of magnetic nanoparticles concentration in biofluid (blood) under influence of high gradient magnetic field. Journal of Magnetism and Magnetic Materials, 323, 32-38, 2011. 
[9] S.A. Khashan, E.P. Furlani, Coupled Particle-Fluid Transport and Magnetic Separation in Microfluidic Systems with Passive Magnetic Functionality. Journal of Physics D Applied Physics, 46, 125002 (15pp), 2013.

[10] C. Kleinstreure, Two-phase Flow Theory and Application, Taylor \& Francis, New York, 2003.

[11] G.C. Bourantas, E.D. Skouras, V.C. Loukopoulos, G.C. Nikiforidis, Meshfree Point Collocation Schemes for 2D Steady State Incompressible Navier-Stokes Equations in Velocity-Vorticity Formulation for High Values of Reynolds Number. Computer Modeling in Engineering \& Sciences, 59, 31-63, 2010.

[12] B.M. Johnston, P.R. Johnson, S. Corney, D. Kilpatrick, Non-Newtonian blood flow in human right coronary arteries: steady state simulations. Journal of Biomechanics, 37, 709-720, 2004.

[13] G.C. Bourantas, V.C. Loukopoulos, E.D. Skouras, V.N. Burganos, G.C. Nikiforidis, "An IPOT Meshless Method Using DC PSE Approximation for Fluid Flow Equations in 2D and 3D Geometries", $13^{\text {th }}$ International Conference of Numerical Analysis and Applied Mathematics 2015 (ICNAAM 2015), Rodos, Greece, September 23-29, AIP Conference Proceedings, 2015. 